\title{
A Recursive Construction of the Regular Exceptional Graphs with Least Eigenvalue -2
}

\author{
I. Barbedo, D. M. Cardoso, D. Cvetković, P. Rama, S.K. Simić*
}

\begin{abstract}
.
In spectral graph theory a graph with least eigenvalue -2 is exceptional if it is connected, has least eigenvalue greater than or equal to -2 , and it is not a generalized line graph. A $(\kappa, \tau)$-regular set $S$ of a graph is a vertex subset, inducing a $\kappa$-regular subgraph such that every vertex not in $S$ has $\tau$ neighbors in $S$. We present a recursive construction of all regular exceptional graphs as successive extensions by regular sets.
\end{abstract}

Mathematics Subject Classification (2010). Primary 05C50; Secondary 06A06.

Keywords. Spectral graph theory, exceptional graphs, posets.

\section{Introduction}

Let $G=(V(G), E(G))$ be a simple graph, where $V(G)$ denotes the nonempty set of vertices and $E(G)$ the set of edges. It is assumed that $G$ is of order $n$, i.e. $|V(G)|=n$. An edge of $E(G)$, which has the vertices $i$ and $j$ as end-vertices is denoted by $i j$. When there is an edge between the vertices $i$ and $j$ we say that these vertices are adjacent. The neighborhood of a vertex $u \in V(G), N_{G}(u)$, is the set of vertices adjacent to $u$, that is, $N_{G}(u)=\{v \in V(G): u v \in E(G)\}$. The degree of vertex $u$ is the cardinality of its neighborhood. A graph $G$ is $r$-regular (or regular of degree $r$ ) if each vertex of $G$ has the same degree $r$.

Throughout the paper, $A_{G}$ denotes the adjacency matrix of $G$, that is $A_{G}=$ $\left(a_{i j}\right)_{n \times n}$, where $a_{i j}=1$ if $i j \in E(G)$ and $a_{i j}=0$ otherwise. The eigenvalues of the graph $G$ are the eigenvalues of its adjacency matrix, here ordered such that $\lambda_{1} \geq \lambda_{2} \geq \cdots \geq \lambda_{n}$. A detailed treatment of graph eigenvalues can be found in $[6]$.

*The authors I. Barbedo, D. M. Cardoso and P. Rama were partially supported by Portuguese funds through the CIDMA - Center for Research and Development in Mathematics and Applications, and the Portuguese Foundation for Science and Technology ("FCT-Fundação para a Ciência e a Tecnologia"), within project PEst-OE/MAT/UI4106/2014. 
A pair $(X, \mathcal{B})$ is a $1-(v, l, \lambda)$ design, if $X$ is a set with cardinality $v$ and $\mathcal{B}$ is a family of $b$ subsets of $X$ with cardinality $l$, called blocks, and each element $x \in X$ lies in exactly $\lambda$ blocks. The incidence matrix $B$ of a $1-(v, l, \lambda)$ design is the $v \times b$ matrix with $i j$-entry equal to 1 if $x_{i} \in B_{j}$ and 0 otherwise. Alternatively, a $1-(v, l, \lambda)$ design $(X, \mathcal{B})$ can be represented by a semi-regular bipartite graph with parameters $(v, b, \lambda, l)$, i.e. by a bipartite graph with $v$ vertices of degree $\lambda$ in one colour class, and $b$ vertices of degree $l$ in another colour class.

A connected graph with least eigenvalue greater than or equal to -2 is either a generalized line graph (with line graphs included), or an exceptional graph (see, e.g., [9]). According to [9, Proposition 1.1.9], a regular connected generalized line graph (see definition, e.g., in [9, Section 1.1]) is either a line graph or a cocktail party graph (a regular graph on $2 k$ vertices of degree $2 k-2$ ). A graph is said to be exceptional if it is connected, has least eigenvalue greater than or equal to -2 , and it is not a generalized line graph. It is known [8] that an exceptional graph has at most 36 vertices, with vertex degrees at most 28 . There are exactly 187 regular exceptional graphs. They are divided into three subsets (to be defined later) called layers. These graphs can be constructed $[1,7,9]$ using different techniques. A comprehensive survey of problems on graphs with least eigenvalue at least -2 , including exceptional graphs, can be found in [9].

A vertex subset $S \subseteq V(G)$ of the graph $G$ is a stable (or independent) set if no pair of vertices in $S$ is connected by an edge. A stable set with maximum cardinality is a maximum stable set.

Given a graph $G$, a partition $\pi=\left(V_{1}, \ldots, V_{r}\right)$ of the vertex set of $G$ is an equitable partition, if for any pair $i, j \in\{1, \ldots, r\}$ there exists $d_{i j} \in \mathbb{N}_{0}$ such that for all $v \in V_{i} d_{i j}=\left|N_{G}(v) \cap V_{j}\right|$, that is, the number of neighbors that a vertex of $V_{i}$ has in $V_{j}$ is independent of the choice of the vertex in $V_{i}$.

A $(\kappa, \tau)$-regular set $S$ of a graph $G$ is a vertex subset which induces a $\kappa$-regular subgraph such that every vertex not in $S$ has $\tau$ neighbors in $S$. If $G$ is a regular graph, then a $(\kappa, \tau)$-regular set $S$ defines an equitable bipartition in $G$.

The $(\kappa, \tau)$-regular sets appeared first in [13], under the designation of eigengraphs, and also in [12], in both cases in the context of strongly regular graphs and designs. Later on, the $(\kappa, \tau)$-regular sets were investigated in the general context of arbitrary graphs $[3,4,5]$.

The aim of this paper is to present a recursive construction of all regular exceptional graphs based on the new $(\kappa, \tau)$-extension technique suggested in [2]. It recursively generates the families of regular exceptional graphs along with a partial order relation among them, and this is represented by its Hasse diagram.

In Section 2 we describe the $(\kappa, \tau)$-extension technique of a regular graph $G$ by a regular graph $H$, and also the partial order relation that arises. The process of extending a graph is reduced to the construction of the incidence matrices of a 1-design (or an appropriate bipartite semi-regular graph).

In Section 3 we propose a technique to construct the regular exceptional graphs, by $(0,2)$-extensions in the case of 1 st and 2 nd layer, and by $(1,3)$-extensions in the case of $3 r d$ layer and using the fact that all these graphs admit an equitable 
partition. In each case we describe how to construct the incidence matrices of the 1-design desired, stating some proprieties. In addition, we also propose an algorithm to construct the regular exceptional graphs in each layer.

In Section 4 we describe, in more details, the computational results that were obtained by the algorithm for the three layers.

The Appendix contains for each regular exceptional graph the list of other regular exceptional graphs with a minimal number of vertices in which it is contained as a proper induced subgraph.

\section{Construction of regular graphs by $(\kappa, \tau)$-extensions}

Let $G$ be a $(p-\tau)$-regular graph of order $n_{1}$ (with $\tau>0$ ) and $H$ a $\kappa$-regular graph (with $\kappa<p$ ) of order $n_{2}$. Our aim is to obtain a $p$-regular graph $H \oplus G$, of order $n_{2}+n_{1}$, such that each vertex in $G$ has $\tau$ neighbors in $H$, and each vertex in $H$ has exactly $p-\kappa$ neighbors in $G$ (hence $V(H)$ is a $(\kappa, \tau)$-regular set in $H \oplus G)$. The procedure that generates the graph $H \oplus G$ from $G$ is called a $(\kappa, \tau)$-extension of $G$ of size $n_{2}$. This construction is possible if we can define a family, $\mathcal{S}$, of $n_{1}$ subsets in $V(H)$, called blocks, each of them with cardinality $\tau$, so that each $v \in V(H)$ is in exactly $p-\kappa$ blocks of $\mathcal{S}$, that is, $(V(H), \mathcal{S})$ is a $1-\left(n_{2}, \tau, p-\kappa\right)$ design. Note that there is a $1-\left(n_{2}, \tau, p-\kappa\right)$ design if and only if $\frac{n_{1}}{n_{2}}=\frac{p-\kappa}{\tau}$. Hence the adjacency matrix of $H \oplus G$ is given by

$$
\left[\begin{array}{cc}
A_{H} & B \\
B^{T} & A_{G}
\end{array}\right]
$$

where $A_{H}$ and $A_{G}$ are the adjacency matrices of $H$ and $G$, respectively, and $B$ is the incidence matrix of a $1-\left(n_{2}, \tau, p-\kappa\right)$ design, that is, each column of $B$ is the characteristic vector of a block. Further on, we shall use graph theoretical terminology and consider the corresponding semi-regular bipartite graphs.

The $(\kappa, \tau)$-extension of a regular graph $G$ by a regular graph $H$ to obtain another regular graph $H \oplus G$, can be applied recursively to generate a sequence of regular graphs. Considering the $(p-\tau)$-regular graph $G$ and the $\kappa$-regular graph $H$ described above, starting with $G_{0}=G$, we can generate a set $\mathcal{F}$ of $((p-\tau)+m \tau)$-regular graphs, $G_{m}$, of order $n_{1}+m n_{2}$, where each $G_{m}$ is obtained by a $(\kappa, \tau)$-extension of $G_{m-1}(m \geq 1)$. Consequently, we can define the following partial order relation $\preceq$ on $\mathcal{F}$, or on any set of graphs.

Definition 2.1. Given $\kappa, \tau, s$, if $G, G^{\prime}$ are regular graphs, then $G \preceq G^{\prime}$ if and only if $G^{\prime}$ can be obtained from $G$ by a sequence of zero or more $(\kappa, \tau)$-extensions of size $s$. 


\section{Construction of regular exceptional graphs}

The set of regular exceptional graphs is partitioned into three layers according to degree $r$ and order $n$ [9, Theorem 4.1.5]. Any such graph is in the 1st, 2nd or 3rd layer, if the following holds, respectively:

(i) $n=2(r+2) \leq 28$,

(ii) $n=\frac{3}{2}(r+2) \leq 27$ and $G$ is an induced subgraph of the Schläfli graph,

(iii) $n=\frac{4}{3}(r+2) \leq 16$ and $G$ is an induced subgraph of the Clebsch graph.

There are 163 graphs in the 1st layer, 21 in the 2 nd layer and 3 in the 3rd layer, i.e. 187 in total.

The regular exceptional graphs are completely described in Table A3 in [9, pp. 213-227]. As in [9], regular exceptional graphs are denoted by numbers $1-187$.

Let $\mathcal{L}$ be the set of regular graphs whose least eigenvalue is greater than or equal to -2 . Hence $\mathcal{L}$ includes all regular exceptional graphs. We shall consider subsets $\mathcal{L}_{1}, \mathcal{L}_{2}, \mathcal{L}_{3}$ of $\mathcal{L}$ in which the ratio $\frac{n}{r+2}$ ( $n$ the number of vertices, $r$ the degree) is the same as in layers $1,2,3$, respectively. Hence, each layer is a subset of the corresponding set $\mathcal{L}_{1}, \mathcal{L}_{2}$ or $\mathcal{L}_{3}$.

Let $G$ be a regular graph of degree $r$ and order $n$. Let $\alpha(G)$ be the size of a maximum stable (or independent) set of $G$ and $\lambda_{n}$ its least eigenvalue. Then

$$
\alpha(G) \leq \frac{-n \lambda_{n}}{r-\lambda_{n}} .
$$

This inequality is known as the Hoffman inequality although A.J. Hoffman never published it. For some bibliographical details related to this bound see [2].

In [2] it is noted that in the case of regular exceptional graphs of the 1st and 2nd layers, the Hoffman upper bound is attained and is equal to the cardinality of a maximum stable set, which is 4 and 3, respectively. This observation has an empirical character and is based on an inspection of the regular exceptional graphs.

In fact, the Hoffman bound is attained for a regular graph $G$ if and only if $G$ has a $(0, \tau)$-regular set such that $\tau=-\lambda_{n}$. The necessary condition was proved in [1] (see also [11]) and the sufficient condition was proved in [2]. The following theorem stems from [10].

Theorem 3.1. Let $G$ be an $r$-regular graph and $\lambda_{n}=-\tau$ its least eigenvalue. For any stable set $S$ of size $s$ and characteristic vector $\mathbf{z}$, we have:

$$
s \leq \frac{n \tau}{r+\tau} .
$$

Furthermore, the following are equivalent:

(i) Equality holds.

(ii) $\mathbf{z}$ is a linear combination of a r-eigenvector and a $\lambda_{n}$-eigenvector.

(iii) The bipartite subgraph induced by the partition $\{S, V(G) \backslash S\}$ is semiregular.

(iv) The partition $\{S, V(G) \backslash S\}$ is equitable. 
It is easy to prove that the stability number of regular exceptional graphs from the first layer is 3 or 4 , in the 2 nd layer is 2 or 3 , and in the 3rd layer is 2 . In fact, if $S$ is a maximum stable set of an $r$-regular graph $G$, then

$$
n-\alpha(G)=\left|\bigcup_{i \in S} N_{G}(i)\right| \leq \sum_{i \in S}\left|N_{G}(i)\right|=\alpha(G) r \Rightarrow \alpha(G) \geq \frac{n}{r+1} .
$$

Therefore, taking in account the relation between the order $n$ of the regular exceptional graphs and their regularities in each layer, combining the Hoffman upper bound with the above lower bound, the result follows.

However, in spite of some efforts, we were not able to prove theoretically that the Hoffman upper bound is attained in regular exceptional graphs in the 1st and 2nd layer.

We shall construct all regular exceptional graphs from the first and second layer by $(0,2)$-extensions. For the first layer the $(0,2)$-extension will have size 4 and for the second layer the size is 3 . Applications of $(0,2)$-extensions will create a stable set of the corresponding size in the resulting graph. This stable set will be a maximum stable set by the Hoffman bound and, from this construction, we may conclude that the Hoffman upper bound is attained for all regular exceptional graphs in the 1st and 2nd layer.

The graphs from the 3rd layer will be considered separately and they will be built by $(1,3)$-extensions.

With each type of $(\kappa, \tau)$-extension we consider the corresponding partial order relation $\preceq$.

As observed in [2], we may state the following result.

Theorem 3.2. Regular exceptional graphs are not minimal elements of the posets $\left(\mathcal{L}_{1}, \preceq\right)$ and $\left(\mathcal{L}_{2}, \preceq\right)$.

As we shall see the same holds for $\left(\mathcal{L}_{3}, \preceq\right)$.

Together with $\mathcal{L}_{1}, \mathcal{L}_{2}, \mathcal{L}_{3}$ one can study the posets $\mathcal{E}_{1}, \mathcal{E}_{2}, \mathcal{E}_{3}$ of exceptional graphs from the three layers with corresponding relations $\preceq$. It would be interesting to study the structure of all these posets. The sets $\mathcal{L}_{1}, \mathcal{L}_{2}, \mathcal{L}_{3}$ are infinite while the sets $\mathcal{E}_{1}, \mathcal{E}_{2}, \mathcal{E}_{3}$ contains $163,21,3$ elements respectively.

Throughout the recursive construction, starting from a regular exceptional graph $G_{1}$, with least eigenvalue -2 , every regular graph $G_{i}$, with $i \geq 2$, obtained by $(\kappa, \tau)$-extensions and with least eigenvalue -2 , remains an exceptional graph.

This conclusion is a consequence of the fact that the graph property of being a line graph is a hereditary property. This means that any induced subgraph of a line graph is also a line graph. Hence we have the following proposition.

Proposition 3.3. Let $G$ be a regular graph with least eigenvalue -2 . Let $H$ be a regular induced subgraph of $G$. We have

(i) if $G$ is a line graph then $H$ is also a line graph,

(ii) if $H$ is an exceptional graph then $G$ is also an exceptional graph. 
3.1. Construction of regular exceptional graphs in the 1 st layer by $(0,2)-$ extensions of size 4. The smallest regular exceptional graphs in the first layer are the five graphs $Z_{1}, Z_{2}, Z_{3}, Z_{4}$ and $Z_{5}$ of order 10 and degree 3 , given in Figure 1 (taken from [9, Appendix A.3]).

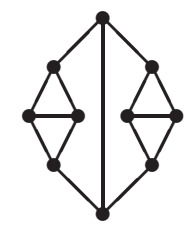

$Z_{1}$

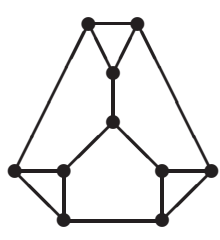

$Z_{2}$

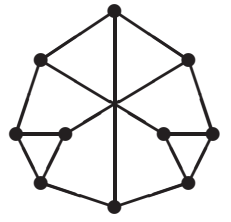

$Z_{3}$

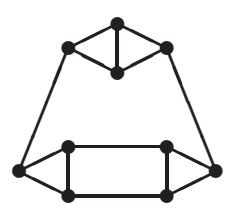

$Z_{4}$

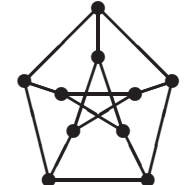

$Z_{5}$

Figure 1. The smallest regular exceptional graphs in the 1st layer.

These graphs are obtained by $(0,2)$-extensions of the graph $3 K_{2}$ of order 6 and regularity 1 , which is a line graph.

The 8 exceptional graphs of order 12 and regularity 4 , are obtained by $(0,2)$ extensions of graphs of order 8 and regularity 2 , that is, one of the three graphs $2 C_{4}, C_{3} \cup C_{5}$ (disjoint union of graphs $C_{3}$ and $C_{5}$ ) or $C_{8}$, which are also line graphs.

Since for regular exceptional graphs of the first layer $r=\frac{n}{2}-2$, with $10 \leq$ $n \leq 28$, in order to build the Hasse diagram of the first layer partially ordered by the relation $\preceq$, this set of exceptional graphs is divided into two partially ordered subsets: the graphs with even regularity, obtained by $(0,2)$-extensions from a graph with even regularity, and the graphs with odd regularity, obtained by $(0,2)$ extensions from a graph with odd regularity.

If $G$ is a graph of even order $n$ and regularity $r=\frac{n}{2}-2$ and $H$ is the 0-regular graph of order 4, the adjacency matrix of the graph $G^{\prime}$ of order $n^{\prime}=4+n$ and regularity $r^{\prime}=\frac{4+n}{2}-2=\frac{n}{2}$ obtained from a graph $G$ by a $(0,2)$-extension is given by

$$
A_{G^{\prime}}=\left[\begin{array}{cc}
O_{4} & B \\
B^{T} & A_{G}
\end{array}\right],
$$

where $O_{4}$ is the adjacency matrix of the graph $H$, that is, the null square matrix of order $4, A_{G}$ is the adjacency matrix of $G$ and $B$ is a matrix with 4 rows and $n$ columns. 
Assuming as a known fact that in the case of regular exceptional graphs of the 1st and 2nd layers, the Hoffman upper bound is attained all these graphs can be constructed by extending graphs with additional 4 or 3 vertices in the way implied by the above considerations.

Let us describe $(0,2)$-extensions of size 4 in some detail.

The graph $G$ is extended by 4 vertices which form a set $S=V(H)$. Let 1, 2, 3,4 be the vertices of $S$. Each vertex of $G$ should become adjacent to exactly two vertices of $S$. Let us define an $r$-regular multigraph $M(S)$ having the set $S$ as the vertex set. If a vertex $v$ of $G$ becomes adjacent to vertices $x, y$ of $S$, then there is an edge labelled $v$ between $x$ and $y$ in $M(S)$. In this way, the vertices of $G$ subdivide the edges of $M(S)$. There are six 2-element subsets of $S$. Constructing $G^{\prime}$ from $G$ by an $(0,2)$-extension means, in fact, to partition the vertex set of $G$ into six subsets which, in turn, should be assigned to 2-element subsets of $S$ in such a way that $M(S)$ is regular of degree $r$. However, the resulting graph $G^{\prime}$ need not to be an $\mathcal{L}$-graph, and this should be checked in the actual constructions.

A multigraph $M(S)$ can be associated with a weighted complete graph on the four vertices of $S$ (see Fig. 2). The weight $x_{i}$ on the $i$-th edge of the multigraph $M(S)$ represents the number of vertices of $G$ adjacent to corresponding vertices in $S$.

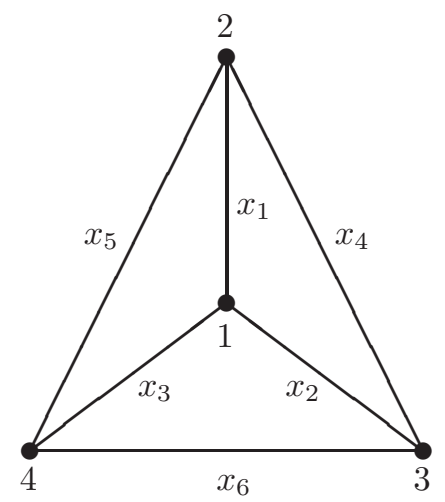

Figure 2. A weighted complete graph with vertex set $\{1,2,3,4\}$.

We have $x_{1}+x_{2}+x_{3}=n / 2$ since this is the degree of 1 in $M(S)$. Hence, $x_{4}+x_{5}+x_{6}=n / 2$. We can conclude that the sum of weights of the edges of any star $K_{1,3}$ and of any triangle $K_{3}$ is equal to $n / 2$. In addition, we have $x_{1}=x_{6}$, $x_{2}=x_{5}$ and $x_{3}=x_{4}$.

Because of automorphisms it is sufficient to consider partitions of the vertex set of $G$ determined by the weights $x_{1}, x_{2}, x_{3}$ with $0 \leq x_{1} \leq x_{2} \leq x_{3} \leq n / 2$. 
Now we shall propose an algorithm to construct the 163 graphs of the first layer by $(0,2)$-extensions which produce equitable partitions.

For a given $n$ construct all feasible triplets $\left(x_{1}, x_{2}, x_{3}\right)$. For each triplet $\left(x_{1}, x_{2}, x_{3}\right)$ we find in turn all ordered partitions of the vertex set of $G$ into parts of cardinalities $x_{1}, x_{2}, \ldots, x_{6}$. For each such partition we consider all graphs $G$ and extend them to a graph $G^{\prime}$ according to this partition. Then, for every graph $G^{\prime}$ we calculate the least eigenvalue and if it is equal to -2 we have generated a regular exceptional graph on $n+4$ vertices. Finally, we should eliminate isomorphic duplicates but record all graph pairs which are in relation $\preceq$.

A suitable computer program based on the above procedure has generated all 163 regular exceptional graph in the first layer. The results are given in the Appendix where for each graph the list of its immediate successors in the poset $\mathcal{L}$ is given.

3.2. Construction of regular exceptional graphs in the 2 nd layer by $(\mathbf{0}, 2)$-extensions of size 3 . Using the procedure of construction by $(\kappa, \tau)$ extensions described in Section 2, our aim is now to build a set of regular graphs obtained by a $(0,2)$-extension, $H \oplus G$, such that $G$ is a $r$-regular graph of order $n$, with $r=\frac{2 n}{3}-2$, and $H$ is the 0 -regular graph of order $|V(H)|=3$. Therefore, this set includes the regular exceptional graphs of the 2 nd layer.

The regular exceptional graphs of the 2nd layer of the lowest order are the graphs of order 9 and regularity 4 in Fig. 3. These graphs are obtained by a $(0,2)-$ extension of the 2-regular graph $C_{6}$ and the disconnected graph $2 K_{3}$, which are line graphs. To construct the Hasse diagram that represents the partially ordered set $\mathcal{E}_{2}$ with relation $\preceq$ of the regular exceptional graphs in the second layer, we consider the $r$-regular graphs of order $n$, with $r=\frac{2 n}{3}-2$ and $6 \leq n \leq 27$.
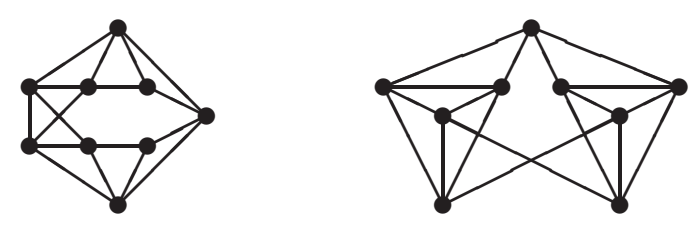

Figure 3. The smallest regular exceptional graphs in the 2nd layer.

In fact, in order to construct each adjacency matrix of the graph $G^{\prime}$, obtained from $G$ by a $(0,2)$-extension, $G^{\prime}=H \oplus G$, it is necessary to determine the incidence matrix with 3 rows and $n$ columns.

Thus, in order to construct the graphs of the second layer we developed an algorithm similar to the algorithm described in the case of graphs from the first layer. The results are given in Appendix. The Hasse diagram of graphs from the second layer is given in Fig. 4. 


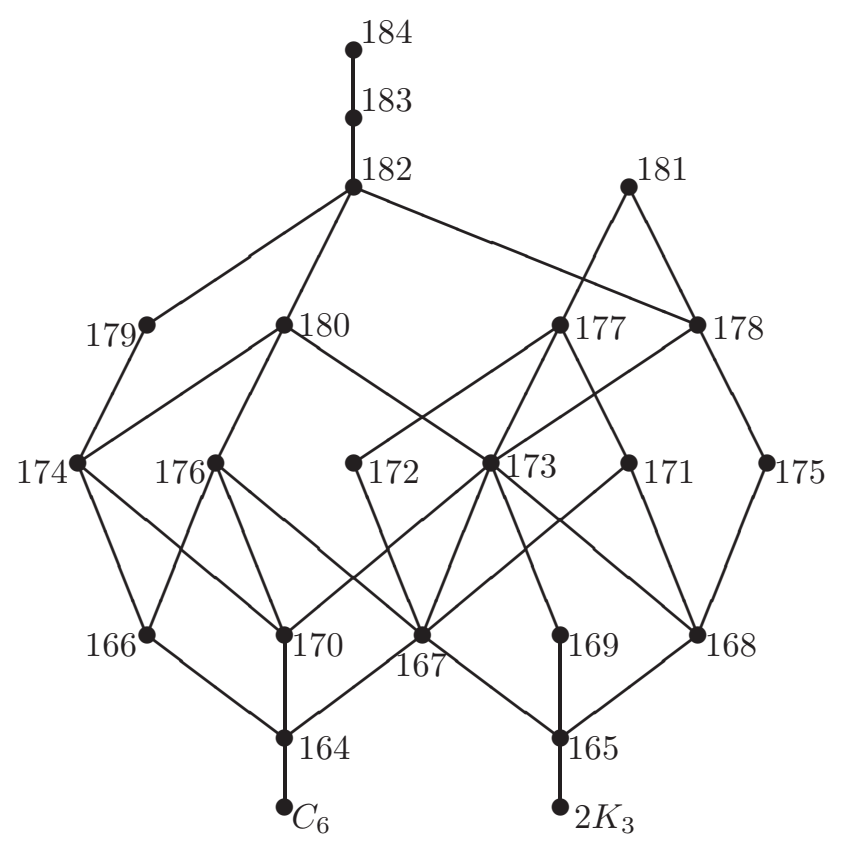

Figure 4. Hasse diagram of graphs from the 2nd layer.

3.3. Construction of regular exceptional graphs in the 3rd layer by $(1,3)$-extensions of size 4 . The exceptional graphs from the 3rd layer are only three. The Hoffman bound is not attained for these graphs. However, it is also possible to build them using the procedure of construction by $(\kappa, \tau)$-extensions described in Section 2, in this case, by (1,3)-extensions of size 4 .

So, the aim now is to build a set of regular graphs obtained by an $(1,3)$ extension, $H \oplus G$, such that $G$ is a $r$-regular graph of order $n$, with $r=\frac{4 n}{3}-2$, and $H$ is the 1-regular graph of order 4,i.e. $2 K_{2}$.

Starting from the line graph $2 K_{2}$ and extending the graphs in turn by four vertex (1,3)-regular sets we obtained the exceptional graphs 185, 186 and 187. They have 8, 12 and 16 vertices respectively. Graph 185 is presented in Fig. 5. Graph 187 is the well known Clebsh graph.

\section{Computational results}

Our algorithm was implemented in Matlab R2009b in order to construct the adjacency matrices of the regular exceptional graphs of the first layer. Similar algorithms were implemented in the cases of the graphs of the second and third layers. The results produced in each layer are described in the Appendix.

The Hasse diagram of the partially ordered set of regular exceptional graphs 


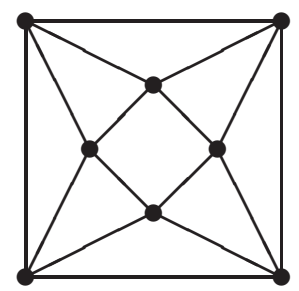

Figure 5. The smallest regular exceptional graph in the 3rd layer.

has four components. Two of these components are associated with the first layer of the regular exceptional graphs: one for the graphs with even degree and the other for the graphs with odd degree.

Notice that there are 3 regular exceptional graphs from the first layer that are not obtained by a $(0,2)$-extension of the minimal graphs $3 K_{2}, 2 C_{4}, C_{3} \dot{\cup} C_{5}, C_{8}$ : the 5 -regular exceptional graph 17 , which is obtained by a $(0,2)$-extension from the disconnected line graph $L_{1}$ (see Fig. 6), the 6-regular exceptional graph 56 , which is obtained by $(0,2)$-extension from the 4-regular line graph $L_{2}$ (see Fig. 6 ) and the 8-regular exceptional graph 134 , which is obtained by $(0,2)$-extension from the 6-regular line graph $L_{3}$ (see the root graph of $L_{3}$ in Fig. 7)

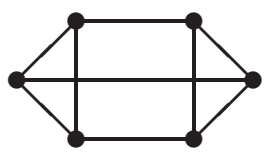

$L_{1}$

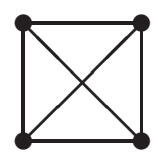

Figure 6. The line graphs $L_{1}$ and $L_{2}$.

The maximal elements in the first layer are the eight regular exceptional graphs of order 20 (113-117, 119-121), all the regular exceptional graphs with order 22 (135-152), and the three Chang graphs (strongly regular exceptional graphs of order 28).

The third component of the Hasse diagram is associated with the regular exceptional graphs from the second layer (see Fig. 4). The minimal elements are $C_{6}$ and $2 K_{3}$ and the maximal elements are the regular exceptional graphs 181 and 184 (the Schläfli graph).

For the third layer there is a minimal graph $2 K_{2}$ and a strongly regular graph, the Clebsh graph, as maximal. 


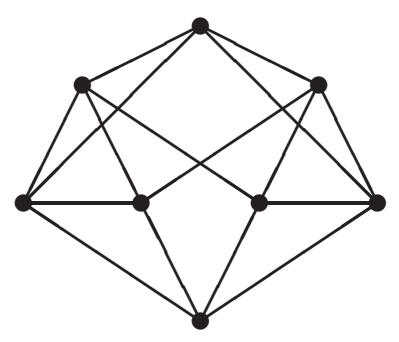

Figure 7. The root graph of the line graph $L_{3}$.

\section{References}

[1] F.C. Bussemaker, D. Cvetković, and J.J. Seidel, Graphs related to exceptional root systems. In Combinatorics, Proc. V Hungarian Colloquium on Combinatorics, Keszthelly $19^{776}$ (ed. A. Hajnal, V. T. Sós), Vol. I, Amsterdam-Oxford-New York 1978, 185-191.

[2] D. M. Cardoso and D. Cvetković, Graphs with least eigenvalue -2 attaining a convex quadratic upper bound for stability number, Bull. Acad. Serbe Sci. Arts, Cl. Sci. Math. Natur., Sci. Math. 133(31) (2006), 41-55.

[3] D. M. Cardoso and P. Rama, Equitable bipartitions of graphs and related results, J. Mathematical Sciences 120(1) (2004), 869-880.

[4] D. M. Cardoso and P. Rama, Spectral results on regular graphs with $(\kappa, \tau)$-regular sets, Discrete Math. 307(11-12) (2007), 1306-1316.

[5] D. M. Cardoso and P. Rama, Spectral results on graphs with regularity constraints, Linear Algebra Appl. 423(1) (2007), 90-98.

[6] D. Cvetković, M. Doob, and H. Sachs, Spectra of Graphs - Theory and Application. Academic Press, New York, 1980.

[7] D. Cvetković, P. Rowlinson, and S. Simić, Graphs with least eigenvalue -2: The star complement technique, J. Algebraic Combinatorics 14(1) (2001), 5-16.

[8] D. Cvetković, M. Lepović, P. Rowlinson, and S. Simić, The maximal exceptional graphs, J. Combinatorial Theory Ser. B, 86(2) (2002), 347-363.

[9] D. Cvetković, P. Rowlinson, and S. Simić, Spectral Generalizations of Line Graphs, on Graphs with Least Eigenvalue -2. Cambridge University Press, Cambridge, 2004.

[10] C.D. Godsil and M.W. Newman, Eigenvalue bounds for independent sets, J. Combinatorial Theory Ser. B, 34(2) (2008), 721-734.

[11] W. Haemers, Interlacing eigenvalues and graphs, Linear Algebra Appl. 226/228 (1995), 593-616.

[12] A. Neumaier, Regular sets and quasi-symmetric 2-designs. In Combinatorial Theory (ed. D. Jungnickel and K. Vedder), Lecture Notes in Mathematics, Vol. 969, Springer, Berlin - Heidelberg 1982, 258-275.

[13] D. M. Thompson, Eigengraphs: constructing strongly regular graphs with block designs, Utilitas Math. 20 (1981), 83-115. 


\section{APPENDIX: Extensions of regular exceptional graphs}

The data on the 187 regular exceptional graphs are given in Table A3 from the book [9]. These graphs are denoted here by numbers $1-187$ and these numbers refer to [9]. The graphs are divided into three layers and into smaller groups according to the number of vertices $n$ and the degree $r$. For each graph the list of regular exceptional graphs obtained by $(\kappa, \tau)$-extensions is given.

First layer

$n=10, \quad r=3$

1. $14,16,18,22,23,24,27,28,30,32,33$

2. $19,20,21,22,23,25,26,27,28,31,32,33,34$

3. $18,27,28,29,30,32,33,34$

4. $15,16,18,19,20,24,25,26,27,28,29,30,31,32,33,34$

5. 30

$n=12, \quad r=4$

6. $37,49,51,57,58,68$

7. $39,40,41,42,46,47,48,51,52,53,55,57,58$

8. $39,40,42,55,57,61,63,64,65,66,67,68$

9. $35,37,44,45,59,60,69$

10. $35,36,40,41,42,47,48,49,53,60,62,64,65$

11. $35,37,38,39,40,46,47,48,49,50,51,54,59,60,62,63,64,67,68$

12. $39,40,46,47,48,49,50,51,52,54,57,58,61,62,63,64$

13. $43,44,45,46,49,51,59,60,67$

$n=14, \quad r=5$

14. $70,83,84,88,89,97,98,99,100$

15. $81,82,90,95,96,105$

16. $84,85,91,92,93,94,97,107$

17. 89,100

18. $90,92,94,97,99,101,103$

19. $71,72,73,74,77,78,86,87,88,90,91,94,95$

20. $71,72,74,78,79,86,88,89,91,92,93,94$

21. $78,88,93$

22. $71,72,74,75,76,80,96,97,99,100,103,104$

23. $71,74,76,77,78,79,97,98,99,101,102,104$

24. $71,72,75,76,81,84,91,92,94,100103,104$

25. $72,73,77,84,87,88,89,97,98,99$

26. 71, 77, 78, 82, 83, 86, 91, 97, 102, 104

27. $71,72,77,78,79,82,84,85,90,93,94,95,96,97,101,102,103$

28. $71,72,74,75,76,78,79,83,84,86,88,92,93,96,97,102,103$

29. $86,90,94,106,107$

30. $88,92,94,95,105,107$ 
31. $77,78,82,86,87,93,97,101,102,107$

32. $71,72,77,79,82,85,90,91,92,93,96,97,101,102,103,105,107$

33. $71,72,75,76,78,79,83,84,86,88,89,91,94,97,98,99,100,102,103$, $104,106,107$

34. $74,78,84,91,94,95,99,102,103,107$

$n=16, \quad r=6$

35. $108,109,111,123,124,126,128,129,132$

36. $108,121,128,131$

37. $110,111,112,123,124,129,130,133$

38. $120,124,128$

39. $115,117,118,119,120,121,122,123,124,126,130,131$

40. $116,117,119,121,123,124,125,126,127,128,129,133$

41. $113,116,121,123,126,127$

42. $114,116,117,120,121,125,128,131,132$

43. $108,109,122,130$

44. $109,112,127,129$

45. 109, 111, 124, 132, 133

46. $113,114,115,116,118,119,122,124,126,127,128$

47. $113,116,117,120,121,124,126,128,131$

48. $114,117,120,121,128$

49. $113,116,119,123,124,127,129,133$

50. $113,114,124,127,129$

51. 114, 115, 116, 118, 123, 124, 127, 129, 130

52. $118,119,121,122,125$

53. $116,117,121,125,131$

54. $114,118,120,125,131$

55. $114,116,117,120$

56. 118

57. $116,117,118$

58. $113,114,115,116,117,118,119$

59. 109, 112, 124, 129, 130, 133

60. 108, 111, 126, 127, 128, 132, 133

61. $114,117,118,119$

62. $113,114,117,119,120$

63. $115,116,118,119$

64. $116,117,120$

65. 128

66. 131

67. $127,130,132$

68. 129

69. 111,112

$n=18, \quad r=7$ 
70. $142,143,146$

71. $136,138,139,140,141,142$

72. $136,138,140,143,144$

73. $135,136,142,145$

74. $138,139,144,145$

75. $136,142,146,147$

76. $136,138,143,147$

77. $137,138,139,141$

78. $139,141,142,143$

79. $138,141,144$

80. 136,146

81. $136,140,149$

82. $137,140,141,152$

83. $138,142,144,147,151$

84. 138, 139, 140, 141, 142, 143, 144, 145, 150, 151

85. $140,141,152$

86. $138,149,151$

87. 139,150

88. $142,150,151$

89. 143,151

90. 137, 140, 148, 149, 150, 152

91. $141,144,149,151,152$

92. $140,149,151$

93. $141,149,150$

94. 140, 145, 149, 150, 151, 152

95. $140,150,152$

96. 136,141

97. 138, 139, 140, 141, 142, 143

98. 138,142

99. $142,143,144,145$

100. $143,144,146,147$

101. 137,141

102. $138,139,141$

103. $140,141,144$

104. $138,141,147$

105. 149,152

106. 151

107. $150,151,152$

$n=20, \quad r=8$

108. $155,156,157$

109. 155,158

110. 153,159

111. $157,158,159,160$ 
112. 158,159

113. -

114. -

115. -

116. -

117. -

118. 155,156

119. -

120. -

121. -

122. $154,155,156$

123. $153,155,156$

124. $155,157,158$

125. 155

126. 156,158

127. 155,158

128. $155,156,157$

129. 153, 158, 159

130. 153, 157, 158

131. 156

132. 155, 157, 160

133. 157, 158, 159

134. 158

$n=22, \quad r=9$

135. - 136. - 137. - 138. - 139. - 140. - 141. - 142. - 143. - 144. - 145. - 146. 147. - 148. - 149. - 150. - 151. - 152. -

$n=24, \quad r=10$

153. 161

154. 162

155. 163

156. 162,163

157. 163

158. 161,163

159. 161

160. 163

$n=28, \quad r=12$

161. - 162. - 163. -

Second layer

$n=9, \quad r=4$

164. 166, 167, 170 
165. 167, 168, 169

$n=12, \quad r=6$

166. 174,176

167. $171,172,173,176$

168. $171,173,175$

169. 173

170. $173,174,176$

$n=15, \quad r=8$

171. 177

172. 177

173. $177,178,180$

174. 179,180

175. 178

176. 180

$n=18, \quad r=10$

177. 181

178. 181,182

179. 182

180. 182

$n=21, \quad r=12$

181. -

182. 183

$n=24, \quad r=14$

183. 184

$n=27, \quad r=16$

184. -

Third layer

$n=8, \quad r=4$

185. 186

$n=12, \quad r=7$

186. 187

$n=16, \quad r=10$

187. - 
Received

I. Barbedo, Center for Research and Development in Mathematics and Applications, EsACT, Politechnic Institute of Bragança, Rua João Maria Sarmento Pimentel, Apartado 128, 5370-326 Mirandela, Portugal.

E-mail: inesb@ipb.pt

D. M. Cardoso, Center for Research and Development in Mathematics and Applications, Department of Mathematics, University of Aveiro, Campus de Santiago, 3810193 Aveiro, Portugal.

E-mail: dcardoso@ua.pt

D. Cvetković, Mathematical Institute, Serbian Academy of Sciences and Arts, Knez Mihailova 26, 11000 Belgrade, Serbia.

E-mail: ecvetkod@kondor.etf.rs

P. Rama, Center for Research and Development in Mathematics and Applications, Department of Mathematics, University of Aveiro, Campus de Santiago, 3810-193 Aveiro, Portugal.

E-mail: prama@ua.pt

S.K. Simić, Mathematical Institute, Serbian Academy of Sciences and Arts, Knez Mihailova 26, 11000 Belgrade, Serbia.

E-mail:sksimic@mi.sanu.ac.rs 